\title{
Load Flow Analysis of a Power System Network in the Presence of Uncertainty using Complex Affine Arithmetic
}

\author{
Yoseph Mekonnen Abebe ${ }^{\mathrm{a}}$, P. Mallikarjuna Rao ${ }^{\mathrm{a}}$, M. Gopichand Nak ${ }^{\mathrm{a}}$ \\ ${ }^{a}$ Dept. of Electrical Eng., College of Engineering (A), Andhra University, Visakhapatnam-530003, India
}

Received: 02 May 2017; Accepted: 17 May 2017; Published: 08 September 2017

\begin{abstract}
The depletion of fossil fuel is driving the world towards the application of renewable energy sources. However, their intermittent nature, in addition to load variation and transmission line sag-tension change due to temperature, is a great deal of problems for reliable power delivery. Without a reliable power delivery, power generation is just a waste of resources. A reliable power delivery can be achieved when the best and the worst case steady state information of a power system network is known to plan and control accordingly. If a system is affected by the presence of uncertainty, a deterministic load flow analysis fails to provide the worst-case load flow result in a single analysis. As a result, a load flow analysis considering the presence of uncertainty is mandatory. On this paper, a novel complex affine arithmetic (AA) based load flow analysis in the presence of generation and load uncertainties is proposed. The proposed approach is tested on an IEEE bus systems and compared with Monte Carlo approach. The proposed approach convergence faster than the Monte Carlo based method and it is slightly conservative.
\end{abstract}

Index Terms: Complex Affine Arithmetic, Monte Carlo Approach, Transmission Line, Renewable Energy Sources, Uncertainty.

(C) 2017 Published by MECS Publisher. Selection and/or peer review under responsibility of the Research Association of Modern Education and Computer Science.

\section{Introduction}

Renewable energy sources, notably the wind and solar energy, are gaining attention due to their long lasting and pollution free nature [1]. Currently, huge numbers of factories are manufacturing solar panel and wind turbines. Though renewable energy sources are abundant and non-exhaustive, the power generated from those sources is not reliable. There is fluctuation in frequency and voltage profile because of frequent variation in

\footnotetext{
* Corresponding author. Tel.: +91 9703758851

E-mail address: yosephgod@gmail.com
} 
weather condition [2]. As a result, the penetration of such renewable energies to the main grid disrupts the voltage profile. Despite their various benefits in terms of global warming, no running cost and naturally abundant, it is difficult for management and planning when a grid is mainly supplied by those sources [3]. The integration of such renewable energies amplifies the imbalance in the grid. This leads to reserve allocation if the exact variation of the generation is precisely predicted [4]. Load forecast plays a very important role for power system planning and decision making [5]. The integration of intermittent energy sources increases the complexity of the power system planning and forecast due to their uncertain nature in addition to frequent load variation. Hence, power system network design must be in consideration of the presence of such variability for reliable power delivery and safety of human being [6].

In order to solve such problem, battery energy storage (BESS) system is proposed and implemented in most areas with those sources of energy. However, when the share of renewable energy source is higher than the fossil fuel in large power grids, the capacity of the battery needed must be high. In today technology, the manufacturing of long lasting battery development does not reach its peak of expectation [7]. Although there is a challenge in renewable energy sources, next generations of grid undeniably consist of both solar and wind power generators. Existing power networks are not constructed in consideration of the integration of intermittent generation sources. Power grids, mostly, distribution systems face the variation of voltage and frequency. There are no means of getting a reliable power from intermittent generation sources. Although operation planning is effective for grid without variable sources, it is difficult to precisely plan and forecasts when there exists a variable generator in the grid. This is mainly due to difficulty in forecasting solar radiation and wind speed even in less weather varying areas [3].

The traditional AGC (Automatic Generation Control) control mechanism for wind turbines and solar panels containing grid is not effective. Due to the intermittent nature of the two energy sources, wind speed and solar insolation, the uncertainty between the actual and the forecasted generation is high. As a result, the energy imbalance increases [8].

Maintaining the energy balance between generation and demand from few seconds to few hours is the key criteria to secure the power system from transient instability. In a place, which is not suitable for hydropower plant, using a backup generation system from fossil fuel as a spinning reserve may reduce the environmental benefits renewable energy sources brings. In such area using energy storage mechanism is preferable than the use of fossil fuels [9].

As a result of the aforementioned variability of generation and the usual load variations, the result of punctual power flow analysis is not well suited for the worst case scenario [10-11]. Therefore, there arises a need for power flow simulation and study that considers the uncertain nature of the aforementioned plants to decrease the complexity of system operation and planning [12]. In order to plan the future loads and generations of a system with intermittent sources, a prominent power flow calculation must be applied which deals with the associated uncertainty.

A robust and reliable power flow analysis is required for optimization, state estimation, voltage control, service restoration, planning, and future expansion of any power systems [10]. A self-validated algorithm based on interval arithmetic (IA) and affine arithmetic (AA) has been proposed to effectively handle power uncertainty by different researchers. A lot of work has been done on the application of IA for power flow analysis with data uncertainty. IA takes into consideration the uncertainties in the system and gives strict bound [11]. But, AA on the other hand gives a conservative bound because of its consideration of round-off and truncation error, besides input uncertainty, better than IA [13], [14]. Both IA and AA based analysis are validated by comparing the result with probabilistic Monte Carlo approach. AA is the extension of IA which overcomes the problems associated with IA by considering round-off and truncation errors beside input errors [15]-[17]. Despite their advantage, a stochastic approach like Monte Carlo suffers from underestimation and deterministic approach like IA suffers from critical estimation.

AA is better suited for the analysis of a true worst-case in circuit tolerance with parameters associated with large uncertainties [18]. In [10] AA based algorithms for power flow analysis in the presence of data 
uncertainties considering sensitivity analysis, in order to determine the partial deviation of the voltage, is presented. If input conditions are uncertain, many conditions need to be analyzed to cover the required range of uncertainty. In such conditions, reliable solution algorithms that take care of the effect of data uncertainty into the power flow analysis are necessary. To address such problem, a new solution methodology based on AA is proposed in [13] and tested on an IEEE 57 bus system. The outcome confirms the conservative nature of AA over MC approach. This is mainly due to Monte Carlo approach does not consider round-off and truncation errors better than AA [10], [13], [14].

The AA based power flow analysis has been done by researchers so far is based on sensitivity analysis which adds an extra burden to the computation. Besides, the Newton-Raphson method needs high orders of Chebyshev approximation in order to represent the trigonometric functions [10], [13], [14], [19]. A GaussSeidel algorithm is used in this paper to solve the growth of non-affine operations, which does not need sensitivity analysis to start the simulation and free from higher order Chebyshev approximation.

This paper mainly focuses on the application of complex AA to deal with uncertainties in power flow analysis. The organization of the paper is as follows: Section 2 deals with the principles of complex AA. Section 3 focuses on complex AA based load flow analysis. Section 4 is dedicated for result and discussion of the proposed load flow analysis. Finally, section 5 presents the conclusion of the overall work.

\section{Complex Affine Arithmetic}

Even though IA has been playing a major role in range analysis, it suffers from dependency problems. As a result, another mechanism, which can address such a problem, is needed and AA is invented. Sources of error during manipulation may be external to the system like less precise and missing input data or a mathematical model that is uncertain by itself or it can be internal to the system. Truncation and round off errors are categorized under internal errors [17]. AA is first formalized and introduced in 1993 by Comba and Stolfi as a basic tool to deal with both internal and external sources of errors and other shortcomings of IA [16, 17, 20]. Although IA solves a lot of problems associated with fixed-point calculation, its dependency problem is high. Dependency problem is simply defined as, having a different result for the same equation with different expressions. Since AA keeps the correlation between computed and input variables and exploits it at each operation, the dependency issue is not a problem. Due to all the aforementioned advantages, AA is better than IAin dealing with uncertain system or function. Most application that has been done with interval arithmetic can be done with AA ones the algorithm is formulated [16, 18]. Like any algebraic function, AA functions have a set of rules governing them to give the desired result. Any number is an element of either a real number or a complex number. The general representation of a complex AA function is given by (1).

$$
\hat{y}^{c}=y_{0}^{c}+\sum_{i=1}^{n} y_{i}^{c} \varepsilon_{i}
$$

From (1) $\hat{y}^{c}$ represents a complex affine function, $y_{0}$ represents the complex central value, $y_{i}$ represents the complex partial deviation and $\varepsilon_{\mathrm{i}}$ represents the noise (symbolic) variable whose value is bounded by $[-1,1]$ interval $[15,17]$.

Let us take two complex affine functions in order to show the mathematical formulas, which govern complex affine manipulation [21]. Equation (2) shows the representation of two complex affine functions. 


$$
\left.\begin{array}{l}
\hat{k}^{c}=k_{0}^{c}+\sum_{i=1}^{n} k_{i}^{c} \varepsilon_{i} \\
\hat{l}^{c}=l_{0}^{c}+\sum_{i=1}^{n} l_{i}^{c} \varepsilon_{i}
\end{array}\right\}
$$

The complex affine function in (2) can be converted into complex IA form according to (3) and become as shown in (4). The reverse, complex interval to complex affine conversion is given by (5).

$$
\left.\begin{array}{l}
\underline{k}_{r}=\operatorname{Re}\left(k_{0}^{c}\right)-\sum_{i=1}^{n}\left|\operatorname{Re}\left(k_{i}^{c}\right)\right| \\
\bar{k}_{r}=\operatorname{Re}\left(k_{0}^{c}\right)+\sum_{i=1}^{n}\left|\operatorname{Re}\left(k_{i}^{c}\right)\right| \\
\underline{k}_{\mathrm{Im}}=\operatorname{Im}\left(k_{0}^{c}\right)-\sum_{i=1}^{n}\left|\operatorname{Im}\left(k_{i}^{c}\right)\right| \\
\bar{k}_{\mathrm{Im}}=\operatorname{Im}\left(k_{0}^{c}\right)+\sum_{i=1}^{n}\left|\operatorname{Im}\left(k_{i}^{c}\right)\right| \\
K=\left[\underline{k}_{r}+j \underline{k}_{\operatorname{Im}}, \bar{k}_{r}+j \bar{k}_{\mathrm{Im}}\right] \mid \\
k_{0}^{c}=\frac{\left(\underline{k}_{r}+\bar{k}_{r}\right)+j\left(\underline{k}_{\operatorname{Im}}+\bar{k}_{\mathrm{Im}}\right)}{2} \\
k_{i}^{c}=\frac{\left(\bar{k}_{r}-\underline{k}_{r}\right)+j\left(\bar{k}_{\mathrm{Im}}-\underline{k}_{\operatorname{Im}}\right)}{2} \\
\hat{k}^{c}=k_{0}^{c}+k_{i}^{c} \varepsilon_{i}
\end{array}\right\}
$$

The basic affine operations based on affine functions in (2) and constant complex number $\psi^{c}$ is given by (6). Equation (6) represents the basic affine equations, which can also be used to formulate non-affine operations.

$$
\begin{aligned}
& \hat{k}^{c} \pm \hat{l}^{c}=\left(k_{0}^{c} \pm l_{0}^{c}\right)+\sum_{i=1}^{n}\left(k_{i}^{c} \pm l_{i}\right) \varepsilon_{i} \\
& \psi^{c} \hat{l}^{c}=\left(\psi^{c} l_{0}^{c}\right)+\sum_{i=1}^{n}\left(\psi^{c} l_{i}^{c}\right) \varepsilon_{i} \\
& \hat{k}^{c} \pm \psi^{c}=\left(k_{0}^{c} \pm \psi^{c}\right)+\sum_{i=1}^{n} k_{i}^{c} \varepsilon_{i}
\end{aligned}
$$

The two most known non-affine operations on complex affine functions are multiplication and division. 
Multiplication is given by (7) and division is given by (8).

$$
\hat{k}^{c} \hat{l}^{c}=k_{0}^{c} l_{c}^{c}+\sum_{i=1}^{n}\left(k_{i}^{c} l_{0}^{c}+l_{i}^{c} k_{0}^{c}\right) \varepsilon_{i}+\left(\sum_{i=1}^{n} l_{i}^{c} \sum_{i=1}^{n} k_{i}^{c}\right) \varepsilon_{n+1}
$$

According to [21, 22], complex affine function division is described as the multiplication of the product of the numerator and the complex conjugate of the denominator by the reciprocal of the product of the denominator and its complex conjugate. An improved complex AA division is proposed by [15] and it is shown in $(8)$.

$$
\left.\begin{array}{r}
\frac{\hat{k}^{c}}{\hat{l}^{c}}=\hat{k}_{0}^{c} C+\sum_{i=1}^{n}\left(C \hat{k}_{i}^{c}-\frac{1}{A B} \hat{k}_{0}^{c} \hat{l}_{i}^{c}\right) \varepsilon_{i}+D \hat{k}_{0}^{c} \varepsilon_{n+1}+ \\
{\left[\sum_{i=1}^{n} f\left(\hat{k}_{i}^{c}\right)\right]\left[\sum_{i=1}^{n} f\left(-\frac{1}{A B} \hat{l}_{i}^{c}\right)+f(D)\right] \varepsilon_{n+2}}
\end{array}\right\}
$$

The constants in the above equation and the function ' $\mathrm{f}$ ' ' are defined as follows: For any complex number $\mathrm{z}=\mathrm{a}+\mathrm{jb}$, the function $\mathrm{f}(\mathrm{z})=|\mathrm{a}|+\mathrm{j}|\mathrm{b}|$. Hence, the constants become:

$$
\begin{aligned}
& A=\hat{l}_{0}^{c}-\sum_{i=1}^{n} f\left(\hat{l}_{i}^{c}\right) \\
& B=\hat{l}_{0}^{c}+\sum_{i=1}^{n} f\left(\hat{l}_{i}^{c}\right) \\
& C=\frac{B+A+2 \sqrt{A B}}{2 A B}-\frac{1}{A B} \hat{l}_{0}^{c} \\
& D=\frac{B+A-2 \sqrt{A B}}{2 A B}
\end{aligned}
$$

For any other non-affine operation, the general Chebyshev formulas in (9) can be used to analyze complex affine functions. Chebyshev approximation is developed using linear interpolation. Higher interpolation gives a good result but with computational cost [18]. For a single affine function, the Chebyshev approximation is given by (3.26).

$$
\hat{p}=\alpha \hat{k}+\xi+\delta \varepsilon_{n}
$$

The coefficients in (3.26), $\alpha, \xi$ and $\delta$, can be found from (10) for a bounded affine function $\hat{k}$ in a bounded interval $K=[\underline{k}, \bar{k}]$.

$$
\left.\begin{array}{l}
\alpha=\frac{f(\bar{k})-f(\underline{k})}{\bar{k}-\underline{k}} \\
\alpha u+\xi=\frac{f(u)+r(u)}{2} \\
\delta=\left|\frac{f(u)-r(u)}{2}\right|
\end{array}\right\}
$$


where $u$ is a point inside $K$ with $f^{\prime}(u)=\alpha$ and $r(x)$ is a line passing through $(\underline{k}, f(\underline{k}))$. and $(\bar{k}, f(\bar{k}))$. In order to get the constants of (10), the function under study must be continuous and its firs derivative must exist. Discontinuous functions within the defined boundary limit cannot be approximated using Chebyshev approximation mechanism by interpolation approach.

\section{Complex AA based Load Flow Analysis}

The AA based load flow analysis initially starts by finding the central values of a load flow problem using Newton-Raphson algorithm. Applying deterministic load flow analysis at the mid of the uncertain load and generation input results in the central value for uncertainty based analysis. The bus voltage magnitude (V0,i) and the bus voltage angle $(\delta 0, \mathrm{i})$ found from the deterministic load flow analysis are the initial parameters for the complex AA based load flow analysis. For any percent of uncertainty "p" in load and generation system of a given power system network, (11) and (12) gives the initial bus voltage magnitude and angle in interval form respectively. Similarly, (13) and (14) give the interval form of both active and reactive power on any bus, respectively. In order to analyze the power system network using complex AA, the interval form of the inputs must be converted to complex AA form using the interval to affine conversion mechanism shown in (5).

$$
\left.\begin{array}{rl}
V_{i}^{I} & =\left[\left(1-\frac{p}{2}\right) V_{0, i},\left(1+\frac{p}{2}\right) V_{0, i}\right] \\
V_{i}^{I} & =\left[\underline{V_{i}}, \bar{V}_{i}\right]
\end{array}\right\}
$$

where $V_{i}^{I}$ represents the bus voltage magnitude in interval form, $\underline{V}_{i}$ represents the lower bound bus voltage magnitude and $\bar{V}_{i}$ represents the upper bound bus voltage magnitude.

$$
\left.\begin{array}{rlr}
\delta_{i}^{I} & =\left[\left(1-\frac{p}{2}\right) \delta_{0, i},\left(1+\frac{p}{2}\right) \delta_{0, i}\right] & \text { if } \delta_{0, i}>0 \\
\delta_{i}^{I}=\left[\left(1+\frac{p}{2}\right) \delta_{0, i},\left(1-\frac{p}{2}\right) \delta_{0, i}\right] & \text { if } \delta_{0, i}<0 \\
\delta_{i}^{I}=\left[\underline{\delta_{i}}, \bar{\delta}_{i}\right] &
\end{array}\right\}
$$

where $\delta_{i}^{I}$ represents the bus voltage angle in interval form, $\delta_{j}$ represents the lower bound bus voltage angle and $\overline{\delta i}$ represents the upper bound bus voltage angle.

$$
\left.\begin{array}{ll}
P_{i}^{I}=\left[\left(1-\frac{p}{2}\right) P_{i},\left(1+\frac{p}{2}\right) P_{i}\right] & \text { if } P_{i}>0 \\
P_{i}^{I}=\left[\left(1+\frac{p}{2}\right) P_{i},\left(1-\frac{p}{2}\right) P_{i}\right] & \text { if } P_{i}<0 \\
P_{i}^{I}=\left[\underline{P_{i}}, \bar{P}_{i}\right] &
\end{array}\right\}
$$

where $P_{i}^{I}$ represents the real power in interval form, $\underline{P}_{i}$ represents the lower bound real power and $\bar{P}_{i}$ represents the upper bound real power. 


$$
\left.\begin{array}{ll}
Q_{i}^{I}=\left[\left(1-\frac{p}{2}\right) Q_{i},\left(1+\frac{p}{2}\right) Q_{i}\right] & \text { if } Q_{i}>0 \\
Q_{i}^{I}=\left[\left(1+\frac{p}{2}\right) P_{i},\left(1-\frac{p}{2}\right) P_{i}\right] & \text { if } Q_{i}<0 \\
Q_{i}^{I}=\left[\underline{Q}_{i}, \bar{Q}_{i}\right] &
\end{array}\right\}
$$

where $Q_{i}^{I}$ represents the reactive power in interval form, $\underline{Q}_{i}$ represents the lower bound reactive power and $\bar{Q}_{i}$ represents the upper bound reactive power.

Using polar to rectangular conversion (11) and (12) merges to result in a complex interval voltage as shown in (15).

$$
V_{i}^{c}=\left[\underline{V}_{i}^{c}, \bar{V}_{i}^{c}\right]
$$

where $V^{c}{ }_{i}$ represents the interval form of complex bus voltage value, $\underline{V}^{c}{ }_{i}$ represents the lower bound of the complex bus voltage value and $\bar{V}_{i}^{c}$ represents the upper bound of the complex bus voltage value

After an interval to an affine conversion of (13-15) the partial deviations of real power, the reactive power, and the voltage become as shown by (16-18) respectively.

$$
\begin{gathered}
{\left[\begin{array}{c}
\hat{P}_{e, 1} \\
\hat{P}_{e, 2} \\
\vdots \\
\vdots \\
\hat{P}_{e, i}
\end{array}\right]=\left[\begin{array}{ccccc}
P_{1,1} & P_{1,2} & \cdots & \cdots & P_{1, i} \\
P_{2,1} & P_{2,2} & \cdots & \cdots & P_{2, i} \\
\vdots & \vdots & \cdots & \cdots & \vdots \\
\vdots & \vdots & \cdots & \cdots & \vdots \\
P_{i, 1} & P_{i, 2} & \cdots & \cdots & P_{i, i}
\end{array}\right]\left[\begin{array}{c}
\varepsilon_{1}^{p} \\
\varepsilon_{2}^{p} \\
\vdots \\
\vdots \\
\hat{Q}_{e, 1} \\
\hat{Q}_{e, 2} \\
\vdots \\
\vdots \\
\hat{Q}_{e, i}
\end{array}\right]=\left[\begin{array}{ccccc}
Q_{1,1} & Q_{1,2} & \cdots & \cdots & Q_{1, i} \\
Q_{2,1} & Q_{2,2} & \cdots & \cdots & Q_{2, i} \\
\vdots & \vdots & \cdots & \cdots & \vdots \\
\vdots & \vdots & \cdots & \cdots & \vdots \\
Q_{j, 1} & Q_{j, 2} & \cdots & \cdots & Q_{i, i}
\end{array}\right]\left[\begin{array}{c}
\varepsilon_{1}^{q} \\
\varepsilon_{2}^{q} \\
\vdots \\
\vdots \\
\varepsilon_{i}^{q}
\end{array}\right]}
\end{gathered}
$$

where $\hat{P}_{e, 1}-\hat{P}_{e, i}$ and $\hat{Q}_{e, 1}-\hat{Q}_{e, i}$ are the total partial deviation power, $P_{1,1}-P_{i, i}$ and $Q_{1,1}-Q_{i, i}$ are the partial deviation coefficients, of the active and reactive power respectively, $\varepsilon^{p}{ }_{1}-\varepsilon^{p}{ }_{i}$ and $\varepsilon^{q}{ }_{1}-\varepsilon^{q}{ }_{i}$ are symbolic variables representing active and reactive power uncertainty respectively.

Since a flat percent of uncertainty " $p$ " is used for both active and reactive power on each bus, the voltage uncertainty, created due to the injection of uncertain power on each bus, initially assumed to equally share both active and reactive power variability [22], as shown by (18).

$$
\hat{V}_{i}^{c}=v_{0, i}^{c}+v_{i}^{c}\left(\varepsilon_{j}^{p}+\varepsilon_{j}^{q}\right)
$$


where $\hat{V}_{i}{ }_{i}$ the affine form of a complex voltage, $V_{0, i}^{c}$ is the central term at bus $i$, and $V_{i}^{c}$ is the complex voltage partial deviation coefficient which is half of the partial deviation of the interval to the affine conversion of (11).

After converting the interval form of complex voltage, real and reactive power to affine forms, the next step is applying a Gauss-Seidel load flow analysis to get the actual values of the bus voltage magnitude and bus voltage angle in the presence of uncertainty. Equation (19) gives the affine form of the first iteration partial deviation voltage. In (19) $Y_{i, i}$ and $Y_{i, k}$ represent the admittance value of the bus " $i$ " and bus " $i$ " with bus " $k$ " respectively for a total of " $n$ " bus. As seen from (19) there are only two non-affine operations created by the division of the complex power by the complex conjugate of the voltage. Applying complex affine operation and non-affine operation and using the inputs in (16-18), the complex AA voltage becomes as shown in (20).

$$
\begin{gathered}
\hat{V}_{e, i}^{c}=\frac{1}{Y_{i, i}}\left[\frac{\hat{P}_{e, i}-j \hat{Q}_{e, i}}{\left(\hat{V}_{i}^{c}\right)^{*}}-\sum_{\substack{k=1 \\
k \neq i}}^{n} Y_{i k} \hat{V}_{k}^{c}\right. \\
{\left[\begin{array}{c}
\hat{V}_{a, 1}^{c} \\
\hat{V}_{a, 2}^{c} \\
\vdots \\
\vdots \\
\vdots \\
\vdots \\
\vdots \\
\hat{V}_{a, i}^{c}
\end{array}\right]=\left[\begin{array}{c}
V_{0,1}^{c} \\
V_{0,2}^{c} \\
\vdots \\
\vdots \\
\vdots \\
\vdots \\
\vdots \\
V_{0, i}^{c}
\end{array}\right]+\left[\begin{array}{cccccccc}
v_{1,1}^{p} & v_{1,2}^{p} & \cdots & v_{1, i}^{p} & v_{1,1}^{q} & v_{1,2}^{q} & \cdots & v_{1, i}^{q} \\
v_{2,1}^{p} & v_{2,2}^{p} & \cdots & v_{2, i}^{p} & v_{2,1}^{q} & v_{2,2}^{q} & \cdots & v_{2, i}^{q} \\
\vdots & \vdots & \vdots & \vdots & \vdots & \vdots & \vdots & \vdots \\
\vdots & \vdots & \vdots & \vdots & \vdots & \vdots & \vdots & \vdots \\
\vdots & \vdots & \vdots & \vdots & \vdots & \vdots & \vdots & \vdots \\
\vdots & \vdots & \vdots & \vdots & \vdots & \vdots & \vdots & \vdots \\
\vdots & \vdots & \vdots & \vdots & \vdots & \vdots & \vdots & \vdots \\
v_{i, 1}^{p} & v_{i, 2}^{p} & \cdots & v_{i, i}^{p} & v_{i, 1}^{q} & v_{i, 2}^{q} & \cdots & v_{i, i}^{q}
\end{array}\right]\left[\begin{array}{c}
\varepsilon_{1}^{p} \\
\varepsilon_{2}^{p} \\
\vdots \\
\varepsilon_{i}^{p} \\
\varepsilon_{1}^{q} \\
\varepsilon_{2}^{q} \\
\vdots \\
\varepsilon_{i}^{q}
\end{array}\right]+\left[\begin{array}{ccc}
v_{1,1}^{a} & \cdots & v_{1, k}^{a} \\
v_{2,1}^{a} & \cdots & v_{2, k}^{a} \\
\vdots & \vdots & \vdots \\
\vdots & \vdots & \vdots \\
\vdots & \vdots & \vdots \\
\vdots & \vdots & \vdots \\
\vdots & \vdots & \vdots \\
\vdots \\
v_{i, 1}^{a} & \cdots & v_{i, k}^{a}
\end{array}\right]\left[\begin{array}{c}
\varepsilon_{1}^{a} \\
\varepsilon_{2}^{a} \\
\vdots \\
\vdots \\
\vdots \\
\vdots \\
\vdots \\
\varepsilon_{k}^{a}
\end{array}\right]}
\end{gathered}
$$

where $\hat{V}_{a, 1^{-}} \hat{V}_{a, i}$ are the new complex AA voltages, $V_{0.1^{-}}^{c} V_{0, i}^{c}$ are the central terms, $V_{1.1^{-}}^{p} V_{i, i}^{p}$ and $V^{q}{ }_{1.1^{-}} V^{q}{ }_{i, i}$ are the partial deviation coefficients for the active and reactive power injections respectively. The terms $\varepsilon^{a}{ }_{1}-\varepsilon^{a}{ }_{k}$ are the new symbolic variables representing approximation errors, $V_{1,1}^{a} V_{i, k}^{a}$ are partial deviation coefficients for the new symbolic variables created due to approximation error.

Since the bus voltage angle can room the whole circle it can be found using the affine to interval conversion. For clarity, it is defined in more prices way as follows.

$$
\begin{aligned}
& \text { let } \hat{z}=(a+j b) \varepsilon_{i} \\
& \text { and } \\
& f(\hat{z})=|a|+j|b|
\end{aligned}
$$

The bus voltage angle in interval form is then:

$$
\begin{aligned}
& \delta_{i}^{I}=\left[\operatorname{angle}\left(V_{0,1}^{c}-\left(\sum_{\substack{i=1 \\
k=1}}^{n} f\left(v_{i, k}^{p}\right)+\sum_{\substack{i=1 \\
k=1}}^{n} f\left(v_{i, k}^{q}\right)+\sum_{i=1}^{n} f\left(v_{i, m}^{a}\right)\right)\right), \text { angle }\left(V_{0,1}^{c}+\left(\sum_{\substack{i=1 \\
k=1}}^{n} f\left(v_{i, k}^{p}\right)+\sum_{\substack{i=1 \\
k=1}}^{n} f\left(v_{i, k}^{q}\right)+\sum_{i=1}^{n} f\left(v_{i, m}^{a}\right)\right)\right)\right] \\
& \quad \text { for } m=1,2
\end{aligned}
$$

Equation (21) is used to find the bus voltage angle for the bus system under study [23]. It is possible to find the bus voltage magnitude in a similar way but the value found should be within the maximum boundary limit 
of the voltage. Unlike bus voltage angle, which can room the whole circle, the bus voltage has a boundary limit [24]. In order to optimize the bus voltage magnitude within the maximum possible limit the first step is separating the partial deviation magnitudes of the complex voltage given by (20) as shown by (22).

$$
\left[\begin{array}{c}
\hat{V}_{1}^{e} \\
\hat{V}_{2}^{e} \\
\vdots \\
\vdots \\
\vdots \\
\vdots \\
\vdots \\
\hat{V}_{i}^{e}
\end{array}\right]=\left[\begin{array}{cccccccc}
\left|v_{1,1}^{p}\right| & \left|v_{1,2}^{p}\right| & \cdots & \left|v_{1, i}^{p}\right| & \left|v_{1,1}^{q}\right| & \left|v_{1,2}^{q}\right| & \cdots & \mid v_{1, i}^{q} \\
\left|v_{2,1}^{p}\right| & \left|v_{2,2}^{p}\right| & \cdots & \left|v_{2, i}^{p}\right| & \left|v_{2,1}^{q}\right| & \left|v_{2,2}^{q}\right| & \cdots & \left|v_{2, i}^{q}\right| \\
\vdots & \vdots & \vdots & \vdots & \vdots & \vdots & \vdots & \vdots \\
\vdots & \vdots & \vdots & \vdots & \vdots & \vdots & \vdots & \vdots \\
\vdots & \vdots & \vdots & \vdots & \vdots & \vdots & \vdots & \vdots \\
\vdots & \vdots & \vdots & \vdots & \vdots & \vdots & \vdots & \vdots \\
\vdots & \vdots & \vdots & \vdots & \vdots & \vdots & \vdots & \vdots \\
\left|v_{i, 1}^{p}\right| & \left|v_{i, 2}^{p}\right| & \cdots & \left|v_{i, i}^{p}\right| & \left|v_{i, 1}^{q}\right| & \left|v_{i, 2}^{q}\right| & \cdots & \left|v_{i, i}^{q}\right|
\end{array}\right]\left[\begin{array}{c}
\varepsilon_{1}^{p} \\
\varepsilon_{2}^{p} \\
\vdots \\
\varepsilon_{j}^{p} \\
\varepsilon_{1}^{q} \\
\varepsilon_{2}^{q} \\
\vdots \\
\varepsilon_{i}^{q}
\end{array}\right]+\left[\begin{array}{ccc}
\left|v_{1,1}^{a}\right| & \cdots & \left|v_{1, k}^{a}\right| \\
\left|v_{2,1}^{a}\right| & \cdots & \left|v_{2, k}^{a}\right| \\
\vdots & \vdots & \vdots \\
\vdots & \vdots & \vdots \\
\vdots & \vdots & \vdots \\
\vdots & \vdots & \vdots \\
\vdots & \vdots & \vdots \\
\left|v_{i, 1}^{a}\right| & \cdots & \left|v_{i, k}^{a}\right|
\end{array}\right]\left[\begin{array}{c}
\varepsilon_{1}^{a} \\
\varepsilon_{2}^{a} \\
\vdots \\
\vdots \\
\vdots \\
\vdots \\
\varepsilon_{k}^{a}
\end{array}\right]
$$

where $\hat{V}^{e}{ }_{1-} \hat{V}_{i}^{e}$ represents the affine form of the magnitude of the total partial deviation voltage.

For a power system network to operate, without damaging its equipment's, altering the stable condition of the network and making it insecure, there must be operational limits of the variables participating in the load flow analysis $[22,25]$. Before optimizing (22) let us see the operational limit of the reactive power and bus voltage magnitude.

\subsection{Generator Bus Power Limit}

Before finding the final bus voltage magnitude and bus voltage angle, the reactive power limit violation must be checked first. A bus switching mechanism must be applied whenever a reactive power limit violation is detected [26]. Assume a natural number 1, 2, 3 represents the slack, the generator, and the load buses respectively, $Q_{i, \min }, Q_{i, \max }$, and $Q_{i, \text { cal }}$ represents the minimum, the maximum reactive power limit and the calculated reactive powers respectively. The bus switching mechanism is applied by comparing the lower and upper reactive power limit with the calculated reactive power.

When the calculated reactive power is lower than the reactive power limit, the generator bus is switched to load bus and its reactive power set to the reactive power limit value. Similarly, when the calculated reactive power is greater than the limit value the generator bus is switched to the load bus and the reactive power limit is set to be its reactive power value. The following pseudo code shows how the reactive power limit is checked and a bus switching approach is applied.

$$
\begin{gathered}
\text { if } Q_{i}^{\text {cal }}<Q_{i}^{\min } \\
\text { type }=3 \\
Q_{i}=Q_{i}^{\min } \\
\text { elseif } Q_{i}^{\text {cal }}>Q_{i}^{\max } \\
\text { type }=3 \\
Q_{i}=Q_{i}^{\max } \\
\text { else } \\
\text { type }=2 \\
\text { end }
\end{gathered}
$$




\subsection{Linear Programming based Optimization}

Equation (22) represents a linear constrained bus voltage partial deviation magnitude. As a result, a linear programming based optimization, within the limit of the maximum bus voltage magnitude deviation between the central voltage value of (20) and the bus voltage limit is considered as the radius of optimization [22].

The radius of the error between the maximum voltage limit and the central voltage, for a percent of uncertainty "p" is shown in (23). Equation (23) is the radiuses for the optimization boundary at a zero center. The percent of uncertainty, in the radius of (23), controls the width of the optimization limit [22]. The higher the percent of uncertainty, the higher is the width and vice versa. When the percent of uncertainty is zero, the system is no longer under the effect of uncertainty, the partial deviation result becomes zero, and the load flow result is equal to the central value.

$$
v_{\lim , i}^{e}=\frac{p}{2}\left(\left|V_{\max , i}\right|-\left|V_{0, i}^{c}\right|\right)
$$

where $v_{l i m, i}^{e}$ represents the radius of the voltage and the angle optimization limit, $V_{\max , i}$ represents the maximum possible bus voltage, and $V_{0, i}^{c}$ represents the central voltage value of (20).

The optimization of (22) based on optimization limit of (23) is given by (24). The maximum and the minimum value of the bus voltage deviation found from (24) is added to the magnitude of the central values in (20) as shown in (25).

$\min / \max$

$$
\begin{gathered}
v_{i}^{e} \\
\text { s.t } \quad-v_{\lim , i}^{e} \leq v_{i}^{e} \leq v_{\lim , i}^{e} \\
-1 \leq \varepsilon_{i}^{p} \leq 1 \\
-1 \leq \varepsilon_{i}^{q} \leq 1 \\
-1 \leq \varepsilon_{i}^{a} \leq 1
\end{gathered}
$$

Equation (25) represents the final voltage magnitude. The final bus voltage angle is already found using (21).

$$
V_{i}=\left|V_{0, i}^{c}\right|+\left[\underline{v_{i}^{e}}, \overline{v_{i}^{e}}\right]
$$

where $V_{i}$ the final voltage value, $V_{0, i}^{c}$ the central bus voltage magnitude from (20), $\underline{v}_{i}^{e}$ and $\vec{v}_{i}^{e}$, are found from (24), represents the minimum and the maximum total partial deviations of bus voltage magnitude respectively.

\section{Results and Discussions}

In order to check the validity of the proposed method, an IEEE test buses are used and implemented using a MATLAB simulation tool. The proposed AA approach is implemented using vectorial representation. Each partial deviation coefficients and central values are represented by a vector with a unique address. For comparison purpose, a well-known Monte Carlo approach is simulated for the same inputs. Monte Carlo approach is used since it the best approximation of the correct solution [19]. The Monte Carlo simulation is 
done for 3000 trials in order to get a non-varying boundary results. Less than 3000 trials the Monte Carlo approach results in a varying output each time the code is simulated. This actually is one of the drawbacks of Monte Carlo method while the proposed complex AA based approach converges within limited number of iterations depending on the number of buses. Two cases are considered to show the validity of the proposed method at a different percent of uncertainty.

\section{CASE A}

- An IEEE-30 bus test system is used to show the effectiveness of the proposed method in comparison with the Monte Carlo approach. The generator and load powers are first varied between $\pm 10 \%$ and then again tested for $\pm 20 \%$ total uncertainty. Generator reactive power limit is taken into consideration.

\section{CASE B}

- An IEEE-57 bus test system is used to simulate the proposed method and the Monte Carlo method. A flat $\pm 15 \%$ and $\pm 25 \%$ generator and load power variations are used. Similar to the aforementioned test cases a reactive power limit is taken into consideration.

\subsection{Case A}

Fig. 1 shows the bus voltage magnitude and the bus voltage angle of an IEEE-30 bus system at $10 \%$ of the generator and load uncertainty. In all the graphs, the lower interval value is represented by "LB" and the higher or the upper interval value is represented by "UB.

The complex AA based approach converges in 3 iterations with a convergence time of 3.465 seconds. The Monte Carlo approach takes 3000 trials and converges in 67.342 seconds.

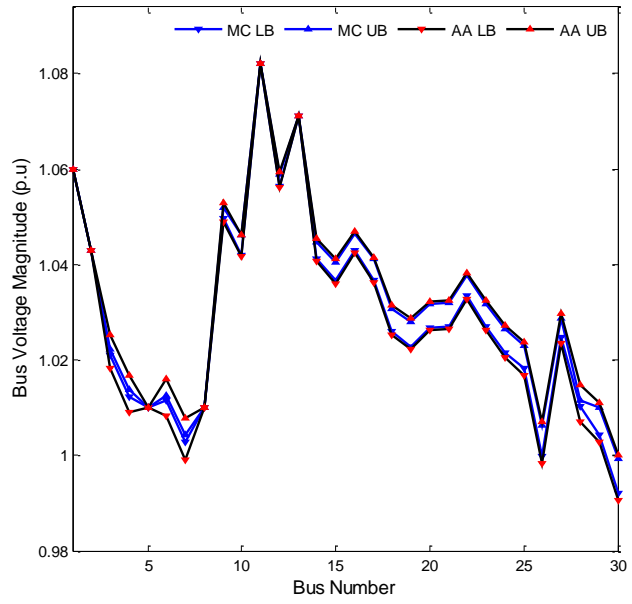

(a)

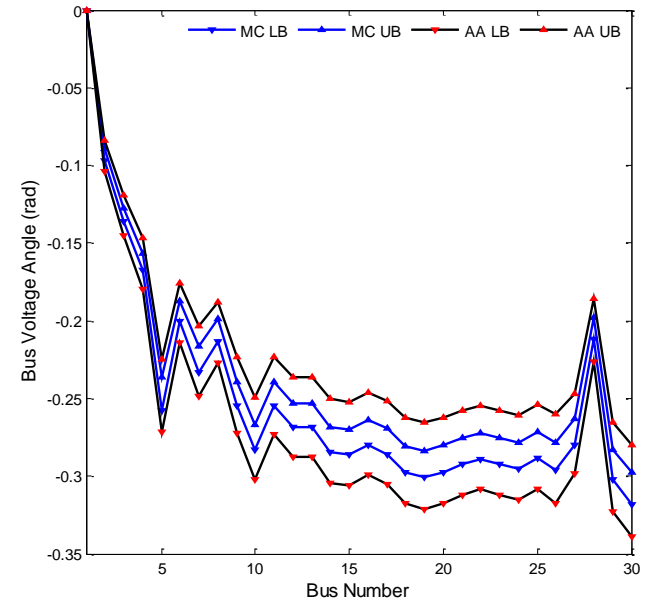

(b)

Fig.1. Case A: at 10\% uncertainty: (a) Bus Voltage Magnitude; (b) Bus Voltage Angle 
In order to further stress, the system a $20 \%$ of generator and load uncertainty is used on an IEEE-30 bus system and the result is depicted in Fig. 2. For the complex AA based approach, it takes 4 iterations and 5.483 seconds to converge while the probabilistic Monte Carlo approach takes 68.029 seconds to give the result of the 3000 trial input values. Moreover, the complex AA based result is conservative than the Monte Carlo based one. This is mainly due to the nature of AA since it takes into consideration the correlation between variables and provides a conservative output than the Monte Carlo approach, which underestimates the worst-case result. When the percent of uncertainty is increased from $10 \%$ to $20 \%$ the number of iteration increases from 3 iteration to 4 iteration respectively. If the number of iteration is different, the time consumption to converge also differs accordingly.

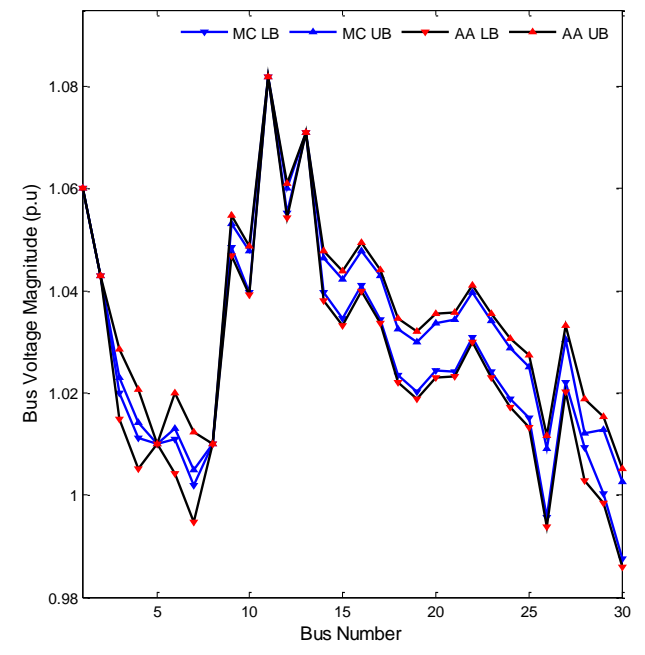

(a)

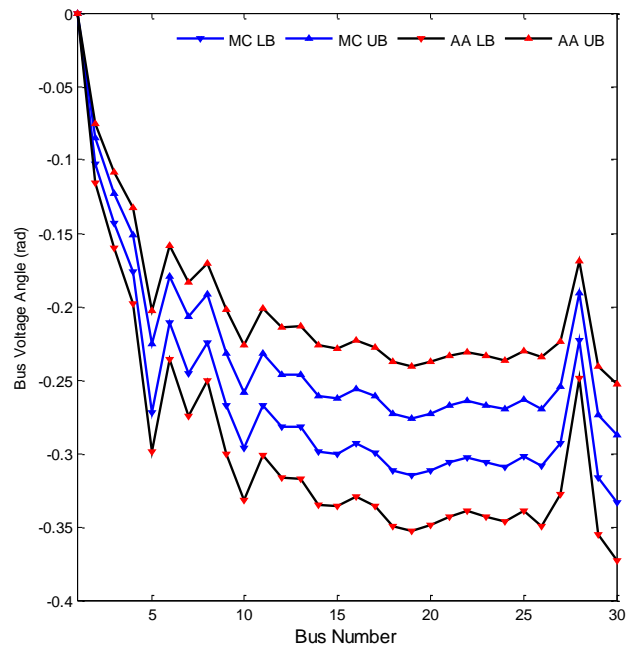

(b)

Fig.2. Case A: at 20\% uncertainty: (a) Bus Voltage Magnitude; (b) Bus Voltage Angle

\subsection{Case B}

Finally, an IEEE-57 bus system is used to validate the proposed method in comparison to the Monte Carlo approach. Fig. 3 shows the bus voltage magnitude and bus voltage angle at $15 \%$ of uncertainty. The complex AA based approach for $15 \%$ of uncertainty converges in 4 iterations at 11.568 seconds while the Monte Carlo based approach takes 243.521 seconds to deal with the 3000 trial input values. Comparing the two results the Monte Carlo approach takes hundreds of seconds to converge while the proposed approach takes few seconds to provide a conservative result.

Stressing the IEEE-57 bus system load and generator power variation to be $25 \%$, results in the bus voltage magnitude and bus voltage angle as shown in Fig 4. Although the wideness of bus voltage angle decreases at some buses, the result shows the complex AA based output voltage and angle are generally conservative when compared to the probabilistic Monte Carlo approach for the specific percent of uncertainty under study. The time it takes for the complex AA base approach to converge is 11.607 second at 4 iterations while the Monte Carlo approach takes 247.513 seconds to converge.

According to the results, when the percent of uncertainty increases, the convergence time increment is very low. The difference of convergence time for $15 \%$ and $25 \%$ of load and generator uncertainty based analysis using the proposed approach is 0.039 second. This indicates that convergence time is less affected by percent of uncertainty when the number of iteration, it take to converge, for both the cases is equal. 


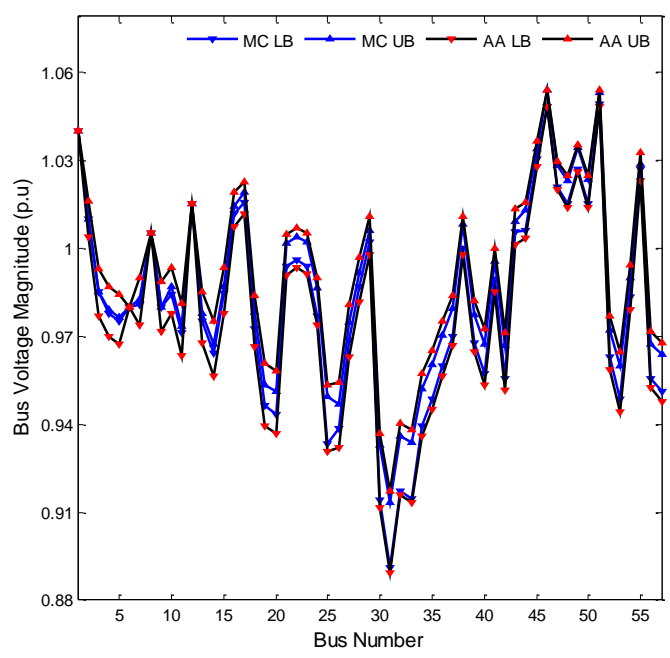

(a)

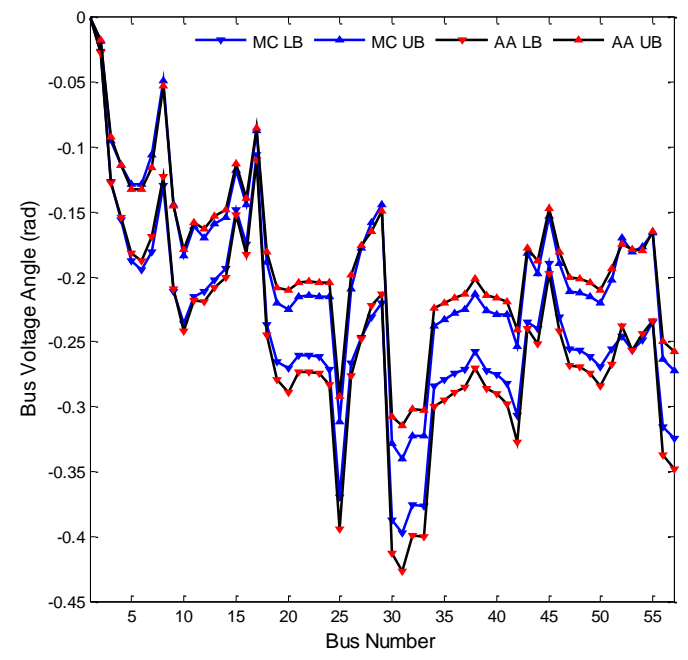

(b)

Fig.3. Case B: at 15\% uncertainty: (a) Bus Voltage Magnitude; (b) Bus Voltage Angle

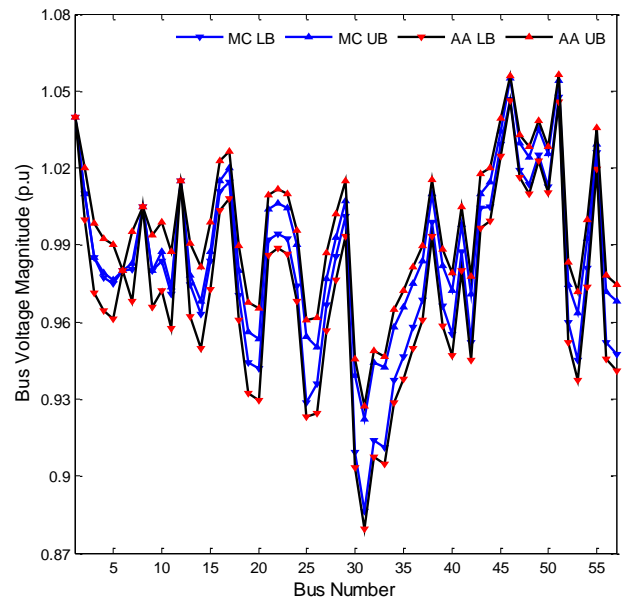

(a)

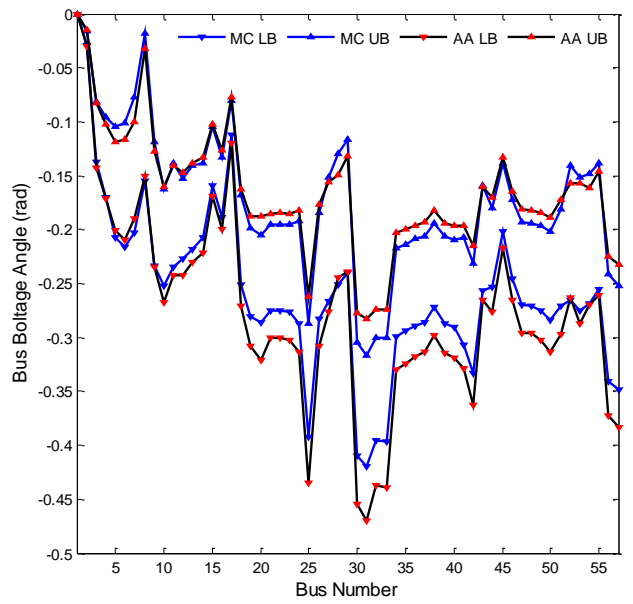

(b)

Fig.4. Case B: at 25\% uncertainty: (a) Bus Voltage Magnitude; (b) Bus Voltage Angle

\subsection{Reactive Power Limit Checking}

Figure 5 and 6 shows the reactive power of the complex AA based result compared to the limit value. Fig 5a and $5 \mathrm{~b}$ represents IEEE-30 bus system calculated reactive power in comparison with the limit value for $10 \%$ and $20 \%$ of uncertainty respectively. Similarly, Fig $6 \mathrm{a}$ and $6 \mathrm{~b}$ represents IEEE-57 bus system calculated reactive power in comparison with the limit value for $15 \%$ and $25 \%$ of uncertainty respectively.

The IEEE-30 bus system for $10 \%$ and $20 \%$ of uncertainty does not violate the reactive power limit and there is no bus switching. On the other hand, the IEEE-57 bus system violates the reactive power limit at bus 2 and 9 for both 15\% and 25\% of uncertainty as shown in Fig 6. The calculated interval reactive power at bus number 2 
and bus number 9 for 15\% of uncertainty is: [-103.4743, -72.7958] MVAr and [-24.8545, -3.5147] respectively. Similarly, for $25 \%$ of uncertainty it is: [-113.0686, -62.2003] MVAr and [-29.8424, 5.9194] respectively. On the other hand, the reactive power limit at bus number 2 and 9 is: [ -17.00, 50.00] MVAr and [-3.00, 9.00] MVAr respectively. Hence, a bus switching from PV to PQ bus is applied to those respective buses and the iteration is repeated until the convergence criterion is achieved.

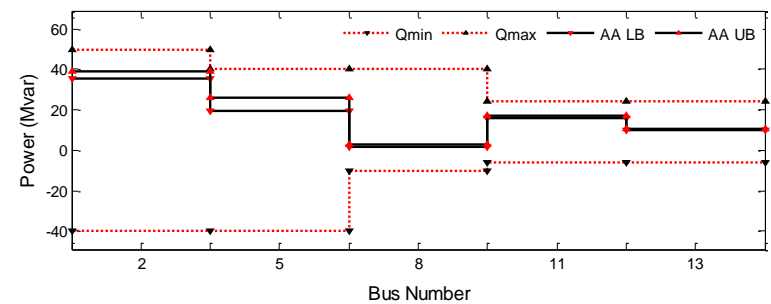

(a)

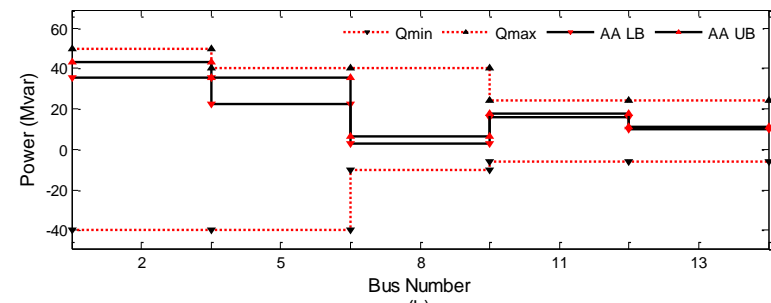

(b)

Fig.5. Case A: Reactive Power Limit: (a) For 10\% of Uncertainty; (b) For 20\% of Uncertainty

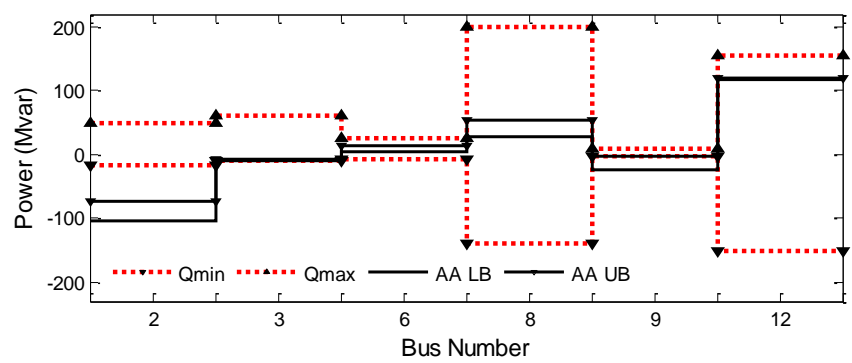

(a)

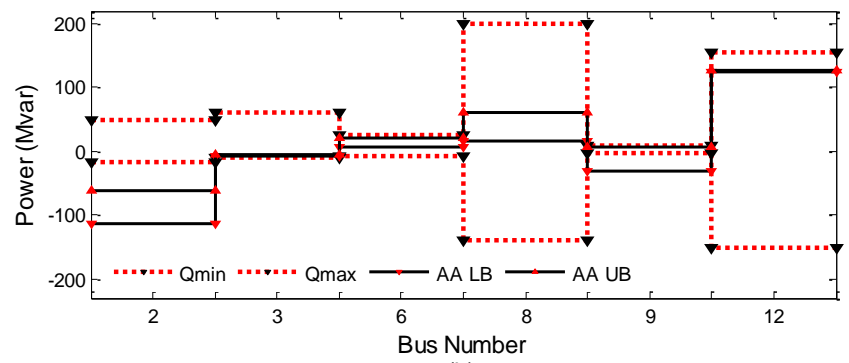

(b)

Fig.6. Case B: Reactive Power Limit: (a) For 15\% Uncertainty; (b) For 25\% of Uncertainty 


\section{Conclusion}

The penetration of variable wind and solar energy, weather change affecting the transmission line parameter, end user load change based on time of the day, unexpected or random disturbances etc. results in an uncertain load and generator data. In order to get the worst-case bus voltage magnitude and bus voltage angle, a complex AA based load flow analysis is proposed and tested on conventional IEEE bus systems. For comparison purpose, a well-known Monte Carlo approach is also simulated and compared with the proposed complex AA based analysis.

A Newton-Raphson based deterministic load flow analysis is used to find the initial bus voltage and angle for the complex AA based analysis and for the analysis of the 3000 Monte Carlo iterations. In order to decrease the number of non-affine operation, created due to the presence of sine and cosine functions in the NewtonRaphson algorithm, a Gauss-Seidel approach is used to find the partial deviations of the complex voltages. The proposed complex AA based load flow algorithm is tested for different percent of uncertainty. In terms of conservatism, fast convergence, and time consumption, the proposed method is better than the Monte Carlo approach. The result found using the proposed approach better represents the worst-case scenario than the probabilistic Monte Carlo approach.

\section{References}

[1] H. Bevrani, A. Ghosh and G. Ledwich, "Renewable energy sources and frequency regulation: survey and new perspectives", IET Renew. Power Gener. IET Renewable Power Generation, vol. 4 no. 5,pp. 438 457, 2010.

[2] K. Tanaka, K. Uchida, K. Ogimi, T. Goya, A. Yona, T. Senjyu, T. Funabashi, and C.-H. Kim, "Optimal Operation by Controllable Loads Based on Smart Grid Topology Considering Insolation Forecasted Error", IEEE Trans. Smart Grid IEEE Transactions on Smart Grid, vol. 2, no. 3, pp. 438-444, 2011.

[3] A. Sobu and G. Wu, "Optimal operation planning method for isolated micro grid considering uncertainties of renewable power generations and load demand", In: proc, IEEE PES Innovative Smart Grid Technologies, pp. 1-6, 2012.

[4] K. D. Vos, J. Morbee, J. Driesen, and R. Belmans, "Impact of wind power on sizing and allocation of reserve requirements," IET Renewable Power Generation, vol. 7, no. 1, pp. 1-9, 2013.

[5] Yixun Shi, "A Probability Model for Occurrences of Large Forest Fires", International Journal of Engineering and Manufacturing (IJEM), vol. 1, no. 1, pp. 1-7, 2012.

[6] Basheer M, Al-Maqaleh, Abduhakeem A, Al-Mansoub, Fuad N and Al-Badani, "Forecasting using Artificial Neural Network and Statistic Models", International Journal of Engineering and Manufacturing (IJEM), vol. 6, no. 3, pp. 20-32, 2016.

[7] G. Capizzi, F. Bonanno, and C. Napoli, "Recurrent neural network-based control strategy for battery energy storage in generation systems with intermittent renewable energy sources", In: proc, 2011 International Conference on Clean Electrical Power (ICCEP), 2011. pp. 336-340, 2011.

[8] L. Gelažanskas, A. Baranauskas, K. A .A Gamage and and M. Ažubalis "Hybrid wind power balance control strategy using thermal power, hydro power and flow batteries, "International Journal of Electrical Power \& Energy Systems", vol 74, pp.310-321, 2016.

[9] A. Lucas and S. Chondrogiannis, "Smart grid energy storage controller for frequency regulation and peak shaving, using a vanadium redox flow battery", International Journal of Electrical Power \& Energy Systems, vol 80, pp.26-36, 2016.

[10] A. Dimitrovski, K. Tomsovic, A. Vaccaro, "Reliable Algorithms for Power Flow Analysis in the Presence of data Uncertainties", In proc, Innovations in Power Systems Reliability pp. 329-357. Springer London., 2011. 
[11] Z. Wang and F.L. Alvarado, "Interval Arithmetic in Power Flow Analysis", IEEE transaction on Power System., vol. 7, no. 3, pp. 1341-1349, 1992.

[12] M. Marin, D. Defour, and F. Milano, "Power Flow Analysis under Uncertainty uses Symmetric Fuzzy Arithmetic", In: Proc, PES General Meeting| Conference \& Exposition, pp. 1-5, Jul. 2014.

[13] A. Vaccaro, C. A. Cańizares, and D. Villacci, "An Affine Arithmetic-Based Methodology for Reliable Power Flow Analysis in the Presence of Data Uncertainty", IEEE Transaction on Power System, vol. 25, no. 2, pp. 624-632, 2010.

[14] M. Pirnia, C. A. Canizares, K. Bhattacharya, and A. Vaccaro, "A Novel Affine Arithmetic Method to Solve Optimal Power Flow Problems With Uncertainties”, IEEE Trans. Power Syst. IEEE Transactions on Power Systems, vol. 29, no. 6, pp. 2775-2783, 2014.

[15] L. H. De Figueiredo, and J. Stolfi, "Affine Arithmetic: concepts and applications", Kluwer Academic Publisher Netherlands, vol. 37, pp. 147-158, 2004.

[16] W. Heindrich, P. Slusallek, H. P. Seidel, "Sampling Procedural Shaders Using Affine Arithmetic", ACM Transactions on Graphics (TOG), vol. 17, no. 3, pp. 158-176, 1998.

[17] J.Stolfi, L.H De Figueiredo, "An Introduction to Affine Arithmetic", Trends in Applied and Computational Mathematics., vol. 4, no. 3, pp. 297-312, 2003.

[18] N. Fermia and G. Spagnuolo, "True Worst-Case Circuit Tolerance Analysis Using Genetic Algorithms and Affine Arithmetic", IEEE Transaction on Circuits and Systems Fundamental Theory and Applications, vol. 47, no. 9, pp. 1285-1296, 2000.

[19] J. Muńoz, C. Cańizares, K. Bhattacharya, A. Vaccaro, "Affine Arithmetic Based Method for Voltage Stability Assessment of Power Systems with Intermittent Generation Sources”, IEEE Transaction on Power System, vol. 28, no. 4, pp. 4475-4487, 2013.

[20] F. Messine and A. Touhami, "A General Reliable Quadratic Form: An Extension of Affine Arithmetic", Reliab. Comput., vol. 12, no. 3, pp. 171-192, 2006.

[21] G. Manson, "Calculating frequency response functions for uncertain systems using complex affine analysis", J. Sound Vib., vol. 288, no. 3, pp. 487-521, 2005.

[22] Y. M. Abebe, M. R. Pasumarthi, and G. N. Mudavath, "Load Flow Analysis of Uncertain Power System Through Affine Arithmetic", In Microelectronics, Electromagnetics and Telecommunications, vol. 372, S. C. Satapathy, N. B. Rao, S. S. Kumar, C. D. Raj, V. M. Rao, and G. V. K. Sarma, Eds. New Delhi: Springer India, 2016, pp. 217-231.

[23] W. Gu, L. Luo, T. Ding, X. Meng, and W. Sheng, "An affine arithmetic-based algorithm for radial distribution system power flow with uncertainties”, Int. J. Electr. Power Energy Syst., vol. 58, pp. 242$245,2014$.

[24] A. Vaccaro, C. A. Canizares, and K. Bhattacharya, "A Range Arithmetic-Based Optimization Model for Power Flow Analysis Under Interval Uncertainty”, IEEE Trans. Power Syst., vol. 28, no. 2, pp. 11791186, 2013.

[25] H. Glavitsch and R. Bacher, "Optimal power flow algorithms", Anal. Control Syst. Tech. Electr. Power Syst., vol. 41, 1991.

[26] S. Mallick, D. V. Rajan, S. S. Thakur, P. Acharjee, and S. P. Ghoshal, "Development of a new algorithm for power flow analysis”, Int. J. Electr. Power Energy Syst., vol. 33, no. 8, pp. 1479-1488, 2011. 


\section{Authors' Profiles}

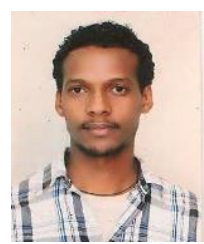

Yoseph Mekonnen Abebe received his MSc in Power System Engineering from Bahir Dar University, Ethiopia in 2013. He is currently a Ph.D. candidate in Andhra University, Visakhapatnam, India. His area of research includes overhead transmission line analysis in the presence of uncertainty, intermittent energy source and their source of variability and load flow analysis in the presence of generation and load uncertainty.

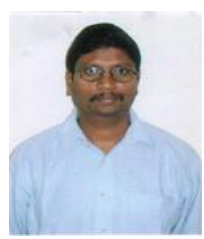

Mallikarjuna Rao Pasumarthi is a Professor of Electrical Engineering in Andhra University College of Engineering Visakhapatnam India. He has decades of teaching experience in the field of electrical engineering in general and control system engineering in particular. His area of research includes design and analysis of specific purpose electrical machines and their controllers, Higher order Model reduction using interval mathematic in the presence of uncertainty and uncertainty based power system analysis.

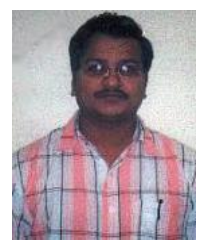

Gopichand Naik Mudavath is an Assistant Professor of Electrical Engineering in Andhra University College of Engineering Visakhapatnam India. His area of research includes high voltage generation, transmission and distribution engineering and an uncertainty based power system analysis.

How to cite this paper: Yoseph Mekonnen Abebe, P. Mallikarjuna Rao, M. Gopichand Nak,"Load Flow Analysis of a Power System Network in the Presence of Uncertainty using Complex Affine Arithmetic", International Journal of Engineering and Manufacturing(IJEM), Vol.7, No.5, pp.48-64, 2017.DOI: 10.5815/ijem.2017.05.05 